\title{
Kinetically Trapped Liquid-State Conformers of a Sodiated Model Peptide Observed in the Gas Phase
}

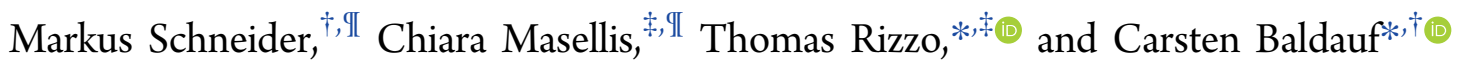 \\ ${ }^{\dagger}$ Theory Department, Fritz-Haber-Institut der Max-Planck-Gesellschaft, Faradayweg 4-6, D-14195 Berlin, Germany \\ ${ }^{\ddagger}$ Laboratoire de Chimie Physique Moléculaire, EPFL SB ISIC LCPM, Ecole Polytechnique Fédérale de Lausanne, Station 6, CH-1015 \\ Lausanne, Switzerland
}

\section{Supporting Information}

ABSTRACT: We investigate the peptide AcPheAla $\mathrm{LysH}^{+}$, a model system for studying helix formation in the gas phase, in order to fully understand the forces that stabilize the helical structure. In particular, we address the question of whether the local fixation of the positive charge at the peptide's C-terminus is a prerequisite for forming helices by replacing the protonated C-terminal Lys residue by Ala and a sodium cation. The combination of gas-phase vibrational spectroscopy of cryogenically cooled ions with molecular
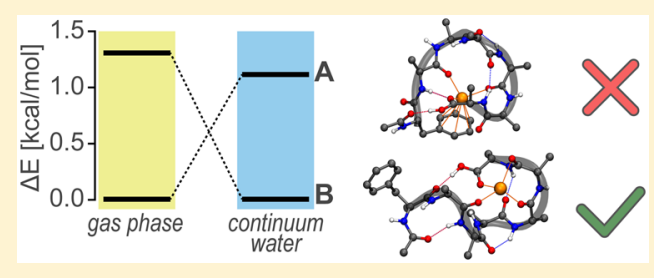
simulations based on density-functional theory (DFT) allows for detailed structure elucidation. For sodiated $\mathrm{AcPheAla}_{6}$, we find globular rather than helical structures, as the mobile positive charge strongly interacts with the peptide backbone and disrupts secondary structure formation. Interestingly, the global minimum structure from simulation is not present in the experiment. We interpret that this is due to high barriers involved in rearranging the peptide-cation interaction that ultimately result in kinetically trapped structures being observed in the experiment.

\section{INTRODUCTION}

Helical secondary structural motifs, such as $\alpha$ and $3_{10}$, are common in proteins. ${ }^{1}$ In solution, the helix propensity is determined both by intramolecular interactions and proteinsolvent interaction. Gas-phase systems offer the opportunity to study the "undamped" intramolecular interactions that shape peptides, thereby shedding light on intrinsic helix propensities and bonding interactions. Gas-phase helices have been investigated using ion mobility spectrometry ${ }^{2-4}$ and vibrational spectroscopy. $^{5-12}$ The combination of these experimental techniques with molecular simulations based on densityfunctional theory (DFT) allows for structure elucidation, as it helps to interpret experimentally obtained spectra. Moreover, a rigorous experiment-theory comparison allows for the assessment of the accuracy and predictive power of simulation approaches. $^{13}$

Pioneering ion-mobility experiments in the group of Jarrold $^{2,3}$ examined the role of $\mathrm{N}$ - and $\mathrm{C}$-terminal residues on gas-phase helix formation for the sequences $\mathrm{Ala}_{n} \mathrm{H}^{+}$, AcLysAla $\mathrm{H}^{+}$, and $\mathrm{AcAla}_{n} \mathrm{LysH}^{+}$. They concluded that $\mathrm{Ala}_{n} \mathrm{H}^{+}$ and $\mathrm{AcLysAla}_{n} \mathrm{H}^{+}$adopt globular conformations in the gas phase independent of the length of the amino-acid chain while $\mathrm{AcAla}_{n} \mathrm{LysH}^{+}$is helical for $n>8 .{ }^{14}$ The identities of these structures were confirmed by theoretical and experimental vibrational spectroscopy in the work of Rossi et al. ${ }^{10}$ and Schubert et al., ${ }^{12}$ respectively. Similar studies focused on peptides of the form $\mathrm{AcPheAla}_{n} \mathrm{LysH}^{+}$with $n=1-5,10$, where phenylalanine provides a UV chromophore, which allows for conformer-specific IR-UV double resonance spectroscopy. ${ }^{6-9}$ Vibrational signatures of individual conformers add a new dimension to peptide structural analysis beyond the orienta- tionally averaged collisional cross section provided by ion mobility. In these experiments, the number of residues necessary to form a helix was found to be six, ${ }^{8,14}$ but much of the hydrogen bonding pattern responsible for the formation of this motif is already present even with only three residues. ${ }^{9,15}$ In conjunction with computational vibrational spectroscopy based on $\mathrm{DFT},{ }^{8-10,16}$ such spectra allowed for determining detailed molecular structures and critically examining evidence for helix formation of peptides in isolation.

Figure 1a illustrates the helix-stabilizing factors in polyalanine peptides, shown for the specific case of $\mathrm{AcPheAla}_{5} \mathrm{LysH}^{+}$. Work by the groups of Jarrold, ${ }^{2,3}$ Rizzo, $^{6-9}$ and Blum ${ }^{12}$ showed that intramolecular hydrogen bonds play an important role and that the design concept can even be transferred to non-natural peptides. ${ }^{11}$ Hoffmann et al. ${ }^{17}$ could show that deleting a single hydrogen bond had little impact on the overall helix stability. In addition to their energetic stability, hydrogen bonds are aligned in helices, and the resulting macro-dipole favorably interacts with the positive charge of the protonated lysine (Lys) sidechain at the C-terminus. Moreover, the capping of the "dangling" carbonyl groups near the C-terminus by the Lys side-chain provides additional stability.

To investigate the importance of the charge fixed at the Cterminus, we focus on the well-studied system $^{8,16}$ of AcPheAla $\mathrm{LysH}^{+}$and compare it to $\mathrm{AcPheAla}_{6}+\mathrm{Na}^{+}$(Figure 1 , parts $b$ and $c$, respectively). In the latter, Lys is formally replaced by alanine $(\mathrm{Ala})$ and a sodium cation $\left(\mathrm{Na}^{+}\right)$in order

Received: June 30, 2017

Revised: August 22, 2017

Published: August 23, 2017 
a) Stabilizing factors of a helix

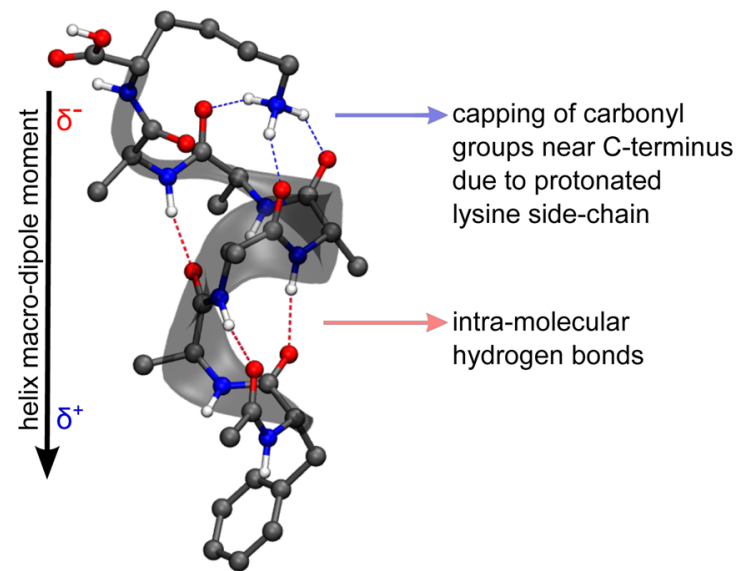

b) AcPheAla ${ }_{5} \mathrm{LysH}^{+}$<smiles>CC(=O)NC(Cc1ccccc1)C(=O)NC(C)C(=O)NC(C)C(=O)NC(C)C(=O)NC(C)C(=O)NC(C)C(=O)NC(CCCCN)C(=O)O</smiles>

c) $\mathrm{AcPheAla}_{6}+\mathrm{Na}^{+}$<smiles>CC(=O)NC(Cc1ccccc1)C(=O)NC(C)C(=O)NC(C)C(=O)NC(C)C(=O)NC(C)C(=O)NC(C)C(=O)NC(C)C(=O)O</smiles>

Figure 1. Illustration of helix-stabilizing factors for peptides in the gas phase (a) and structural formulas of (b) $\mathrm{AcPheAla}_{5} \mathrm{LysH}^{+}$and $_{(\mathrm{c})} \mathrm{AcPheAla}_{6}+$ $\mathrm{Na}^{+}$.

to introduce a freely movable positive charge. The resulting rich possibilities for electrostatic interaction can locally disrupt hydrogen-bonding networks and induce unconventional backbone conformations. ${ }^{18-21}$ Consequently, the cation-binding site, and hence the conformation as a whole, is not a priori obvious. Ion mobility studies on metalated peptides (e.g., sodiated species of $\mathrm{Ala}_{n}+\mathrm{M}^{+22}$ ) suggest that the cation plays the same role as the charged Lys side-chain in $\mathrm{AcAla}_{n} \mathrm{LysH}^{+}$for peptides with $n>12$. For shorter peptides, calculated collisional cross sections (CCS) for globular and helical structures are both in agreement with the experimental CCS, preventing a definitive structural assignment. In the present work, we couple IR-UV double resonance spectroscopy and theory in order to unravel the structure of the system of $\mathrm{AcPheAla}_{6}+\mathrm{Na}^{+}$with the aim of understanding whether a freely movable cation is sufficient to stabilize helix formation or if the C-terminal localization is a prerequisite for that.

\section{EXPERIMENTAL SETUP}

The experimental setup has been described in detail elsewhere. ${ }^{23}$ In brief, a nanoelectrospray ion source is combined with a cooled ion trap $(4 \mathrm{~K})$ for spectroscopic studies of gasphase ions. Conformer-selective IR spectra are recorded by applying IR-UV double resonance. A measurement is performed by fixing the wavenumber of the UV laser to a line in the electronic spectrum and scanning the wavenumber of an infrared laser. When the IR pulse is in resonance with a vibrational transition of the ion, part of the population is removed from the ground state, leading to a decrease in UVinduced fragmentation. Scanning the IR wavenumber, one obtains a conformer-specific vibrational spectrum. Performing the same experiment on each line of the electronic spectrum allows for assignment of each UV spectral feature to a particular conformer.

\section{COMPUTATIONAL METHODS}

The applied conformational search algorithm is similar to the one used by Rossi et al. ${ }^{16}$ First, a global conformational search is performed on the force field (FF) level using CHARMM $22^{24}$ and OPLS-AA, ${ }^{25,26}$ separately. To that end, a basin-hopping approach $^{27}$ was applied using the scan program of the TINKER molecular modeling package. ${ }^{28,29}$ For the system of AcPheAla $_{5} \mathrm{LysH}^{+}\left(\right.$AcPheAla $\left._{6}+\mathrm{Na}^{+}\right) 603280$ (626 829) conformers were found using CHARMM22 and 643938 (635 120) conformers were found using OPLS-AA. Singlepoint energy calculations at the generalized-gradient approximated (GGA) DFT level of theory have been performed for all these FF conformers. All DFT calculations were done using the all-electron/full-potential electronic structure code package FHI-aims. ${ }^{30-32}$ To be more precise, energies were computed at the $\mathrm{PBE}+\mathrm{vdW}$ level, i.e. using the $\mathrm{PBE}^{33}$ functional and a pairwise van der Waals correction scheme (vdW). ${ }^{34}$ Furthermore, FHI-aims-specific tier 1 basis sets and light settings have been used that are provided out-of-the-box to control the computational accuracy intended to give reliable energies energy for screening purposes. ${ }^{30}$ For the two FFs individually, the 500 conformers with the lowest FF energy and the 500 conformers with the lowest DFT energy, i.e., a grand total of 2000 conformers, have been selected. The 2000 selected conformers were then geometry optimized at the PBE+vdW level using tier 1 basis sets and light settings. A hierarchical clustering scheme was applied in order to rule out duplicates. Further relaxation was then accomplished at the PBE+vdW level using FHI-aims-specific tier 2 basis sets and tight settings that are intended to provide meV-level accurate energy differences, $^{30}$ i.e. within $0.02 \mathrm{kcal} / \mathrm{mol}$. After clustering, this resulted in 324 (159) conformers for the system of AcPheAla $_{5} \mathrm{LysH}^{+}\left(\mathrm{AcPheAla}_{6}+\mathrm{Na}^{+}\right)$in the low-energy region, i.e., within $6 \mathrm{kcal} / \mathrm{mol}$ from the global minimum. These conformers were then again locally refined at the PBE0+MBD level, i.e., using the hybrid exchange-correlation (xc) functional $\mathrm{PBEO}^{35}$ augmented by a many-body dispersion (MBD) correction, ${ }^{36}$ using tier 2 basis sets and tight settings which resulted in 52 (23) conformers in the low-energy region, i.e., within $3 \mathrm{kcal} / \mathrm{mol}$ from the global minimum.

\section{RESULTS AND DISCUSSION}

AcPheAla ${ }_{5} \mathrm{LysH}^{+}$. For our comparative study, a firm assignment of measured conformer-selective IR spectra to their calculated counterparts is of paramount importance. To that end, we first reassess the peptide $\mathrm{AcPheAla}_{5} \mathrm{LysH}^{+}$and demonstrate that the applied conformational search technique completely grasps the conformational space energetically close to the global minimum, and that the applied level of theory is capable of reproducing the energetics as well as the vibrational properties of the conformers. For this we compare our results 
to previous work on $\mathrm{AcPheAla}_{5} \mathrm{LysH}^{+}$by Stearns et al., ${ }^{8}$ where the 45 lowest-energy structures were selected out of a set of 1000 force-field minima and subsequently optimized using DFT with a hybrid exchange-correlation (xc) functional. Even though four structures were successfully assigned to the experimental spectra, the question whether the search was complete and the whether these conformers are located in the global minimum region remained open. This did, in part, motivate an exhaustive conformational search by Rossi et al., ${ }^{16}$ in which seven conformers were found within $1 \mathrm{kcal} / \mathrm{mol}$ of the global minimum on the potential-energy surface (PES). The authors were able to assign the experimentally observed structures to the global minima populated at low temperature by using the hybrid $x c$-functional PBE0, ${ }^{35}$ augmented by a many-body dispersion (MBD) correction, ${ }^{36}$ and including zeropoint energy corrections. The latter were computed with the generalized-gradient approximation functional $\mathrm{PBE}^{33}$ and a pairwise van der Waals correction (vdW), ${ }^{34}$ which proved however unsatisfying for the prediction of vibrational spectra. It was suggested that using a hybrid $\mathrm{xc}$-functional was necessary, which was a natural assumption since this level of theory was necessary for a correct conformational energy prediction in the first place. Furthermore, it was assumed that an anharmonic treatment was needed to yield improved spectra.

The conformational search strategy has already been laid out in detail in the previous section, including numbers illustrating the exhaustiveness of the search. The fact that we find two additional conformers within $1 \mathrm{kcal} / \mathrm{mol}$ from the lowestenergy conformer gives us confidence in the conformational search. The corresponding hierarchy of the relative DFT energy $\Delta E$ on the PES is shown in Figure 2. Nine conformers were found within $1 \mathrm{kcal} / \mathrm{mol}$ from the global minimum.

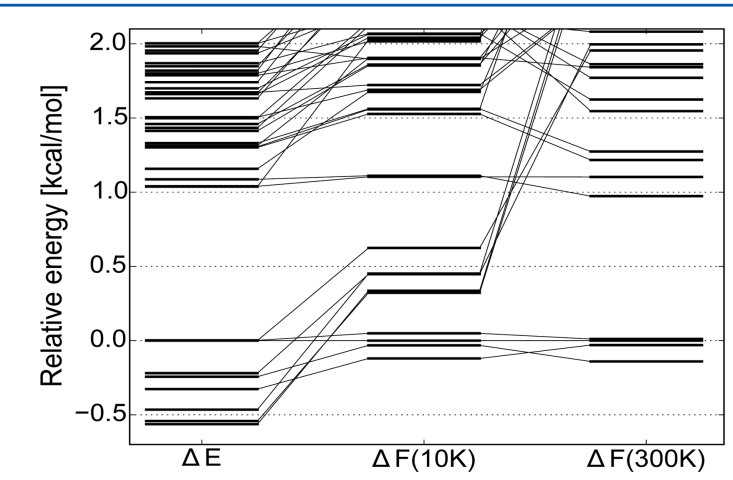

Figure 2. Energy hierarchies of conformers of $\mathrm{AcPheAla}_{5} \mathrm{LysH}^{+}$at the PBE0+MBD energy $\Delta E$ as well as the Helmholtz free energy $\Delta F$ at 10 and $300 \mathrm{~K}$ with harmonic vibrational free energy contributions calculated at the PBE+vdW level.

Since the experimental measurement takes place on cold ions in the gas phase, the PES merely allows for a rough estimate about the structures populated at low temperatures. To confidently assign the experimentally observed structures one needs to rely on the Helmholtz free energy $F$ at $10 \mathrm{~K}$, as this is approximately the temperature of the observed ions. We account for free energy contributions from internal degrees of freedom, consisting of vibrations and rotations, in addition to the DFT energy $E$ on the PES. A detailed formulaic description is provided in the Supporting Information. For $\mathrm{AcPheAla}_{5} \mathrm{LysH}^{+}$, Figure 2 shows energy hierarchies of the $\mathrm{PBE} 0+\mathrm{MBD}$ energy $\Delta E$ as well as the Helmholtz free energy
$\Delta F$ at $10 \mathrm{~K}$ and at $300 \mathrm{~K}$, always relative to conformer $\mathbf{A}$ (see Figure $3 \mathrm{~b}$ ). At this stage, harmonic vibrational free energy contributions have been calculated at the PBE+vdW level. While the $\Delta F(10 \mathrm{~K})$ surface should best resemble experimental conditions of gas-phase measurements at $10 \mathrm{~K}$, the free energy hierarchy at $300 \mathrm{~K}$ represents an estimate of the conformers populated at the early stage of the experimental process, where the molecules are electrosprayed into the instrument at room temperature. Their low free energy at $10 \mathrm{~K}$ and the relatively large gap to alternative structures at $300 \mathrm{~K}$ indicate why the species observed in experiment should be among the four conformers within $0.25 \mathrm{kcal} / \mathrm{mol}$ from the global minimum. Of course we are aware of the limitation of not taking into account anharmonicity and the possibility of solvation-memory effects (i.e., kinetic trapping).

High computational costs prohibited the systematic use of hybrid xc-functionals for the calculation of harmonic vibrations in the previous study by Rossi et al. ${ }^{16}$ To complete the picture, we repeat the harmonic vibrational free energy calculations at the PBE0+MBD level, confirming the already obtained result. Figure 3a shows the energy hierarchies for $\Delta E, \Delta F(10 \mathrm{~K})$, and $\Delta F(300 \mathrm{~K})$ for the four lowest-energy conformers illustrated in Figure $3 \mathrm{~b}$. Conformers $\mathbf{A}$ and $\mathbf{B}$ are virtually identical near the C-terminus, but differ near the $\mathrm{N}$-terminus by a tilted Phe side chain. The difference between conformers $\mathbf{C}$ and $\mathbf{D}$ is similar. All four conformers show helical structure motifs: conformer C possesses one $31_{10^{-}}$and two $\alpha$-helical turns, conformer $\mathbf{D}$ features one $3_{10^{-}}$and one $\alpha$-helical turn, and conformers $\mathbf{A}$ and B each possess two $3_{10^{-}}$and one $\alpha$ turn.

For this work, the original IR-UV double resonance experiment by Stearns et al. $^{8}$ has been repeated to allow conformer-selective IR spectra to be compared to their theoretical counterparts calculated at the PBE0+MBD level. The affiliated UV spectrum including peak assignments to their corresponding conformers is provided in the Supporting Information. The conformer-selective IR spectra are shown in Figure 3c. Conformers $\mathbf{A}$ and $\mathbf{B}$ could be attributed to their corresponding observed IR spectra. While the agreement is very good, the match between experimental and theoretical IR spectra is not perfect. This discrepancy is commonly attributed to two factors: (i) the effect of a possible incomplete characterization of electron exchange and correlation, despite the use of the hybrid functional PBE0, and (ii) the treatment of anharmonic vibrations and nuclear quantum effects. ${ }^{37}$ Both of these effects are corrected for solely by applying a scale factor to the vibrational frequencies. The assumption of a uniform overestimation of the harmonic vibrational modes with respect to experiment is debatable as they depend on the theoretical method, the used basis set, and the system itself. ${ }^{38,39}$ In this work, we focus on the frequency region of 3200 to $3500 \mathrm{~cm}^{-1}$ which is sensitive to $\mathrm{N}-\mathrm{H} \cdots \mathrm{O}$ hydrogen bonding, where a uniform scaling factor of 0.948 yields very good agreement.

The exhaustive conformational search presented here for $\mathrm{AcPheAla}_{5} \mathrm{LysH}^{+}$, and the rigorous treatment of harmonic vibrations at the hybrid xc level allowed for (i) reproducing the known energy hierarchy and finding additional conformers in the low-energy region and (ii) calculating well-fitting harmonic IR spectra for the conformers in the low-energy region. In this way we confirm the conformers predicted by Stearns et al. ${ }^{8}$ and Rossi et al. ${ }^{16}$ and can rule out any other competing conformers. This also shows that calculating computationally costly anharmonic IR spectra is not required in this case. Now that we have confirmed the accuracy of our simulation approach, we 


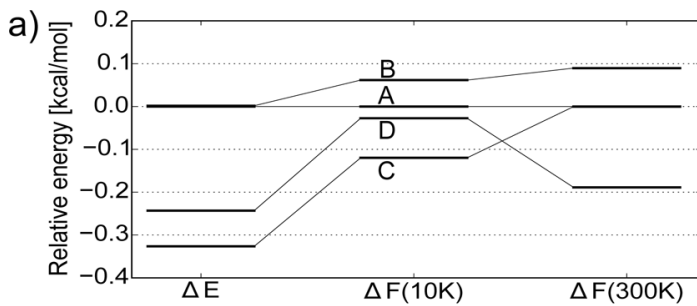

b)
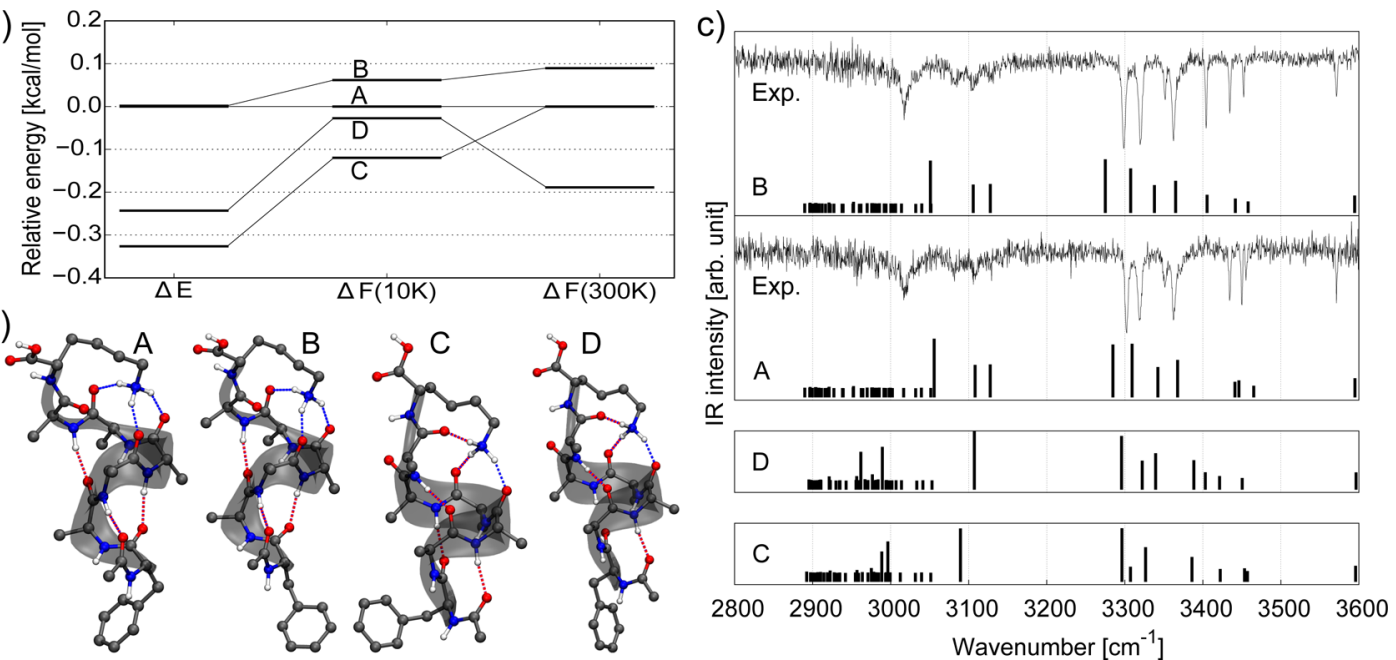

Figure 3. (a) Relative DFT energies $\Delta E$ as well as relative Helmholtz free energies $\Delta F$ at 10 and $300 \mathrm{~K}$ for the lowest-energy conformers of AcPheAla $\mathrm{LysH}^{+}$at the PBE0+MBD level. (b) Four lowest-energy conformers on the $\Delta F(10 \mathrm{~K})$ scale. Hydrogen bonds are indicated with dashed lines. The labeling of the conformers follows Stearns et al. ${ }^{8}$ (c) Two measured conformer-selective IR spectra (traces) are compared to harmonic vibrational calculations (sticks). Calculated spectra are uniformly scaled by a factor of 0.948 .

tackle $\mathrm{AcPheAla}_{6}+\mathrm{Na}^{+}$, a more challenging system because of the additional conformational degrees of freedom due to the "unfixed" cation.

$\mathrm{AcPheAla}_{6}+\mathrm{Na}^{+}$. Figure 4 shows the energy hierarchies of the relative $\mathrm{PBE} 0+\mathrm{MBD}$ energies $\Delta E$ as well as the relative

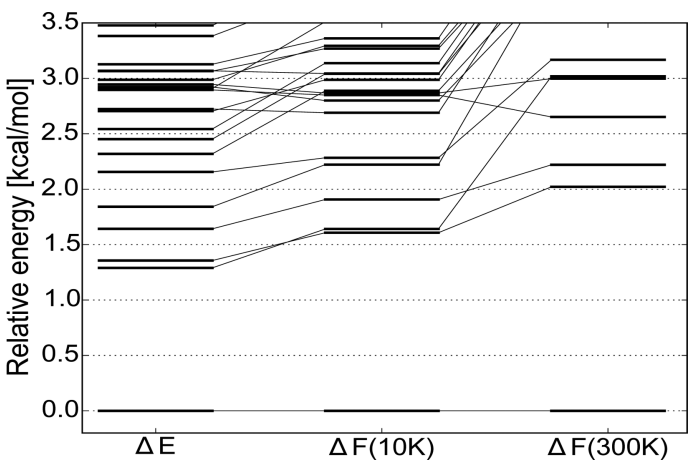

Figure 4. Energy hierarchies of conformers of $\mathrm{AcPheAla}_{6}+\mathrm{Na}^{+}$at the $\mathrm{PBE} 0+\mathrm{MBD}$ energy $\Delta E$ as well as the relative Helmholtz free energy $\Delta F$ at 10 and $300 \mathrm{~K}$ with harmonic vibrational free energy contributions calculated at the $\mathrm{PBE}+\mathrm{vdW}$ level.

Helmholtz free energies $\Delta F$ at 10 and $300 \mathrm{~K}$ with harmonic vibrational free energy contributions at the $\mathrm{PBE}+\mathrm{vdW}$ level that were obtained for $\mathrm{AcPheAla}_{6}+\mathrm{Na}^{+}$. The four presumably dominant conformers are presented in Figure $5 \mathrm{~b}$. Of the four conformer-selective IR spectra that were recorded, two of them correspond to conformers with particularly high intensity in the UV spectrum (see Figure S2, Supporting Information). The measured IR spectra of these two conformers, IIa and IIb, show very good agreement with the IR spectra calculated at the PBE0+MBD, which uses a scale factor of 0.948. Both conformers are nearly identical, differing only in the tilt of the Phe side chain near the $\mathrm{N}$-terminus. They are globular with the peptide being "wrapped around" the $\mathrm{Na}^{+}$cation with four partially negatively charged $\mathrm{C}=\mathrm{O}$ groups pointing toward the positively charged cation, restricting them from forming the hydrogen bonds necessary for helix formation. Indeed, no similarities are observed comparing these structures to the helical motifs of AcPheAla $\mathrm{LysH}^{+}$. The C-terminal fixation of the charge by the Lys side-chain seems to be a prerequisite to effectively cap the helix. The "freely movable" charge prevents helix formation in this system and instead induces a globular motif. All conformers found in the low-energy region (i.e., within $3 \mathrm{kcal} / \mathrm{mol}$ from the global minimum) show a globular conformation.

An obvious observation is the outstanding global minimum (conformer I in Figure $5 \mathrm{~b}$ ) that is separated by a $1.6 \mathrm{kcal} / \mathrm{mol}$ gap from the next minimum on the $\Delta F(10 \mathrm{~K})$ scale. The clear assignment of conformers IIa and IIb to the two most intense bands in the measured spectra suggests that both conformers may be kinetically trapped. Moreover, the most stable structure I does not seem to be observed in the experiment-none of the conformer-selective spectra fit the calculated vibrational signatures (see Figure 5c). The structure representing the global minimum is globular and features a cation- $\pi$ interaction between the $\mathrm{Na}^{+}$and the Phe side chain. If that conformer were present in experiment, one would expect broad features in the UV spectrum due to charge-transfer between $\mathrm{Na}^{+}$and the aromatic ring. However, no such features have been observed. The reason behind the kinetic trapping of conformers IIa and IIb has to be sought in the experimental procedure in which the molecules are electrosprayed into the apparatus from a solution at room temperature while the actual measurements are taken on isolated molecules at $10 \mathrm{~K}$.

The energy landscape of the system may differ significantly between the system in solution at room temperature and in the gas phase at $10 \mathrm{~K}$. This is particularly true for the global minima. While the global minimum in the gas phase may be energetically favored in comparison to the other structures, kinetic constrains, i.e., high energy barriers between minima, may hinder proper folding while transitioning from solution to the gas phase. Thus, experimentally observed local minima in the gas phase higher in energy are yielded due to their structural bias from aqueous solution at room temperature, resulting in kinetically trapped structures unable to transition into the global minimum.

It is obvious from comparing the $\Delta F(10 \mathrm{~K})$ and $\Delta F(300 \mathrm{~K})$ hierarchies (see Figure 5a) that the temperature difference does 

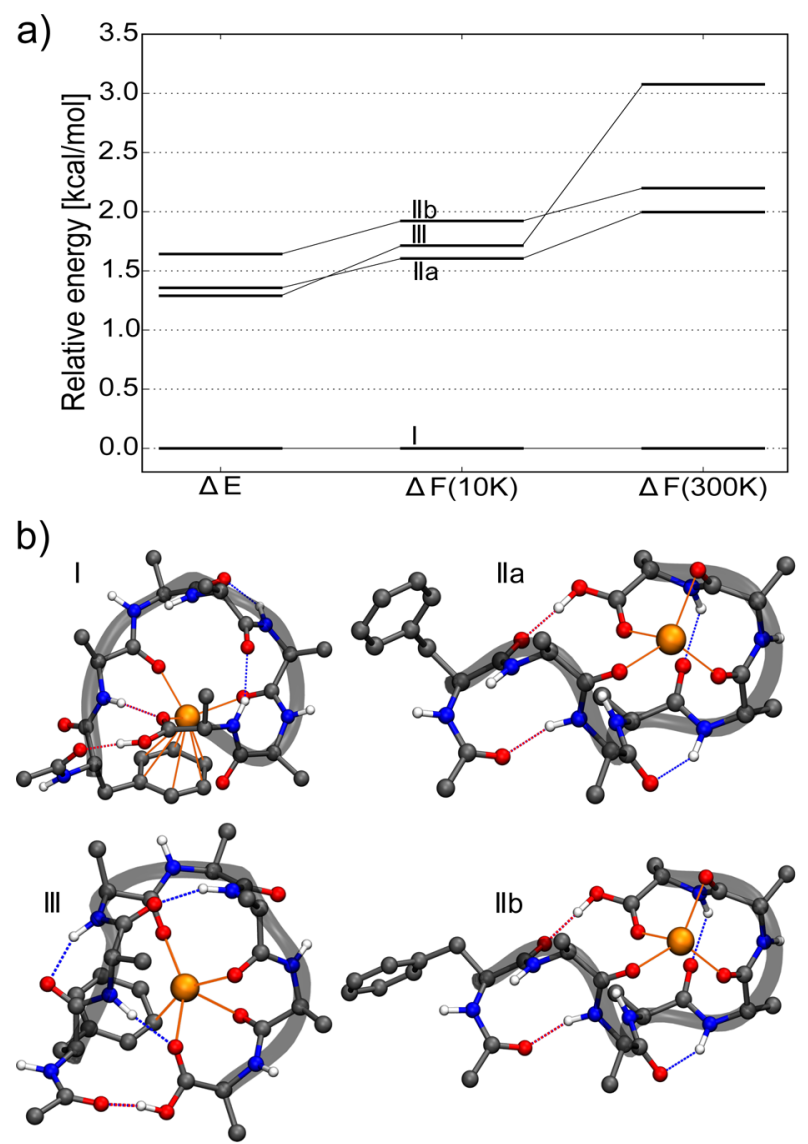

c)
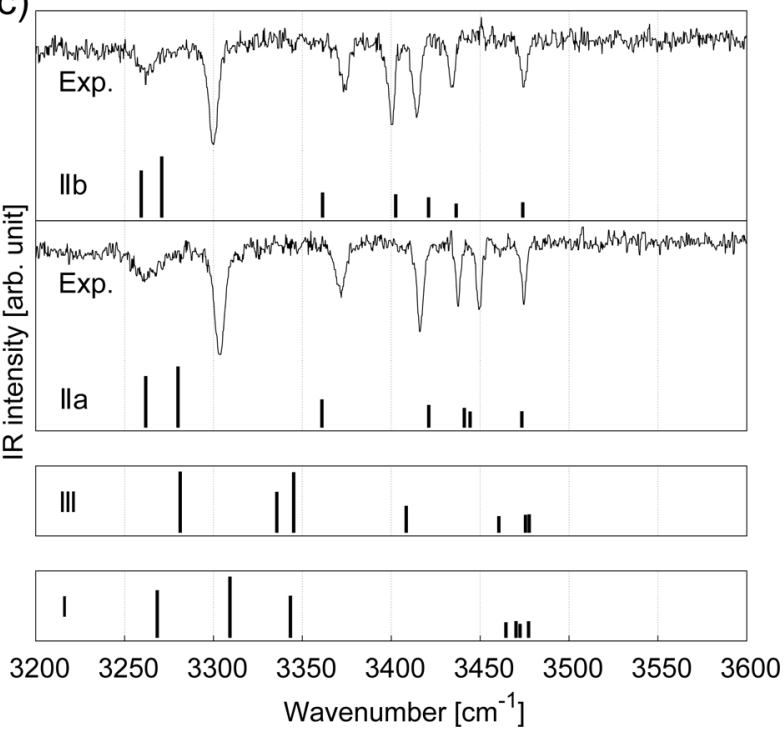

Figure 5. (a) Relative DFT energies $\Delta E$ as well as relative Helmholtz free energies $\Delta F$ at 10 and $300 \mathrm{~K}$ for the lowest-energy conformers of AcPheAla $_{6}+\mathrm{Na}^{+}$at the PBE0+MBD level. (b) Four lowest-energy conformers on the $\Delta F(10 \mathrm{~K})$ scale. Hydrogen bonds are indicated with dashed lines. (c) Two measured conformer-selective IR spectra (traces) with highest intensity are compared to harmonic vibrational calculations (sticks). Calculated spectra were uniformly shifted by a factor 0.948 .

not contribute to a possible kinetic trapping effect. In fact, the energy gap between the global and the next minimum even increases from $1.6 \mathrm{kcal} / \mathrm{mol}$ at $10 \mathrm{~K}$ to $2.0 \mathrm{kcal} / \mathrm{mol}$ at $300 \mathrm{~K}$. Therefore, kinetic trapping must be caused by solvation effects alone. In order to estimate the magnitude of such an effect, the four lowest-energy conformers presented in Figure 5 have been geometrically optimized with $\mathrm{PBE} 0+\mathrm{MBD}$ including implicit water by solving the modified Poisson-Boltzmann (MPB) equation $^{40,41}$ implemented ${ }^{42}$ in FHI-aims (consult the Supporting Information for computational details). While in the gas phase conformer I is $1.6 \mathrm{kcal} / \mathrm{mol}$ lower in DFT energy than the next minima (conformers IIa and IIb), the situation is reversed when including implicit aqueous solution; conformer I is now $0.9 \mathrm{kcal} / \mathrm{mol}$ higher in energy. This suggests that they carry a structural bias from aqueous solution, i.e. the barriers are sufficiently high to kinetically trap them during the electrospray process.

A similar scenario can be seen for conformer III, which is of comparable energy as conformers IIa and IIb on the $\Delta F(10 \mathrm{~K})$ scale, but the calculated IR spectrum, presented in Figure 5c, does not match any experimentally observed one. Consulting the $\Delta F(300 \mathrm{~K})$ scale (see Figure 5a) shows that conformer III is $0.9 \mathrm{kcal} / \mathrm{mol}$ higher in energy than conformer IIb at room temperature. When rerelaxing the structures to the nearest minimum on the potential energy surface at the PBE0+MBD level including implicit aqueous solvation effects as described above, conformer III becomes further energetically penalizedit is then more than $5.0 \mathrm{kcal} / \mathrm{mol}$ higher in energy compared to the other conformers.

There remain two conformers, IV and $\mathbf{V}$, for which the UV spectral signatures have lower intensity (see Figure S2), suggesting that they have smaller populations. The corresponding IR spectra, shown in Figure 6a, could not be assigned to their calculated counterparts for any structure within $6 \mathrm{kcal} /$ mol from the global minimum on the $\Delta F(10 \mathrm{~K})$ scale. Similarly, as for IIa and IIb, we assume that these conformers are kinetically trapped, which also renders their assignment difficult as these conformers might be higher in energy, and thus no energy criterion can be applied for finding them. Instead we follow an approach ${ }^{43}$ where we make use of information from the experiment in order to select from the overall pool of structures for calculation of spectra. Candidates were picked if they feature a free carboxylic acid $\mathrm{OH}$ stretch, since the experimental IR spectra show a peak at $3578 \mathrm{~cm}^{-1}$ (see Figure 6a). Because of the absence of broad features in the UV spectrum, only structures were considered where the $\mathrm{Na}^{+}$ cation was not in close proximity to the phenyl ring. In total, vibrational spectra for 126 conformers have been calculated. In addition to that, local refinement on the PBE0+MBD level for all 52 found minima structures within $3 \mathrm{kcal} / \mathrm{mol}$ from the global minimum for the system of $\mathrm{AcPheAla}_{5} \mathrm{LysH}^{+}$has been laid out after formally replacing Lys with $\mathrm{Ala}+\mathrm{Na}^{+}$, with the sodium cation being placed at the position of the amino group nitrogen. Vibrational spectra for the resulting 28 conformers (after clustering) have been calculated as well. As explained above, computationally costly hybrid xc-functionals are required in order to gain enough accuracy. Only conformer IV (see Figure $6 \mathrm{~b}$ ), lying $13.6 \mathrm{kcal} / \mathrm{mol}$ higher in energy than the global minimum on the $\Delta F(10 \mathrm{~K})$ scale, could be assigned to one of the less populated conformers. However, one peak in the simulated vibrational spectrum is blue-shifted by $80 \mathrm{~cm}^{-1}$ with respect to the nearest experimental peak, and the corresponding vibrational mode is indicated in Figure $6 \mathrm{~b}$ with a green arrow. Conformer IV is a candidate for the kinetically trapped structure only because of the (partially) matching IR spectra. Taking into account the large computational effort taken, a more appropriate and computationally affordable technique for 

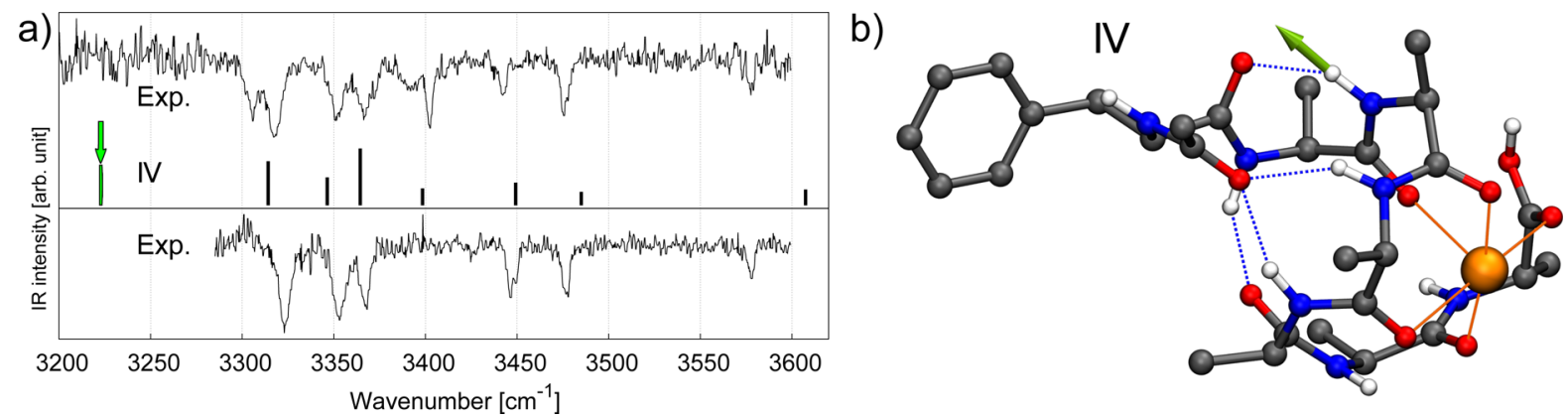

Figure 6. (a) For the system of AcPheAla $6+\mathrm{Na}^{+}$, comparison of two measured conformer-selective IR spectra (traces) with lowest intensity to vibrational calculations (sticks) in harmonic approximation on the PBE0+MBD level for structure IV. Calculated spectra have been shifted by applying a uniform scaling factor of 0.948. (b) Structural form of conformer IV. Hydrogen bonds are indicated with dashed lines. The highlighted vibrational mode in part $a$ is indicated with a green arrow in part $b$.

finding kinetically trapped conformers would be certainly desirable.

\section{CONCLUSION}

Our data indicate that the fixed location of the charge at the Cterminus is imperative for helix formation in peptides of this length in isolation, as this stabilizes the structure through a cation-helix dipole interaction. In the case of the freely movable sodium cation, the cation-backbone and cation $-\pi$ interactions seem to be stronger, leading to local distortions of peptide structure, preventing helix stabilization. It is interesting to note the high barriers that seem to be involved in interconverting one structure to another. Even though the cation $-\pi$ interaction is energetically favored for the AcPheAla $6+\mathrm{Na}^{+}$in the gas phase, the system remains kinetically trapped in a structural state that is characterized by cation-backbone interactions and that is energetically preferred in a polar solvent.

\section{ASSOCIATED CONTENT}

\section{(S Supporting Information}

The Supporting Information is available free of charge on the ACS Publications website at DOI: 10.1021/acs.jpca.7b06431.

Detailed and technical description of the experiment and applied theoretical conformational search methods, observed UV spectra, Helmholtz free energy calculations, and DFT calculations including implicit solvent effects (PDF)

Numerical details for all shown energy hierarchies as well as experimental and calculated IR spectra, i.e., for Figures 2-6 and Figure S4, and the corresponding $x y z$-files of conformers (ZIP)

\section{AUTHOR INFORMATION}

\section{Corresponding Authors}

*(T.R.) E-mail: thomas.rizzo@epfl.ch.

*(C.B.) E-mail: baldauf@fhi-berlin.mpg.de.

\section{ORCID $\odot$}

Thomas Rizzo: 0000-0003-2796-905X

Carsten Baldauf: 0000-0003-2637-6009

\section{Author Contributions}

${ }^{\mathrm{II}}$ Both authors contributed equally.

Notes

The authors declare no competing financial interest.

\section{ACKNOWLEDGMENTS}

The authors thank the joint Max-Planck-EPFL Center for Molecular Nanoscience and Technology for financial support. C.B. thanks Dr. Mariana Rossi for sharing her knowledge about theoretical vibrational spectroscopy and Prof. Matthias Scheffler for his continuous support. The experimental work was supported by the EPFL as well as the Swiss National Science Foundation through Grant 200020_165908.

\section{REFERENCES}

(1) Barlow, D. J.; Thornton, J. M. Helix Geometry in Proteins. J. Mol. Biol. 1988, 201, 601-619.

(2) Hudgins, R. R.; Ratner, M. A.; Jarrold, M. F. Design of Helices That Are Stable in Vacuo. J. Am. Chem. Soc. 1998, 120, 12974-12975.

(3) Hudgins, R. R.; Jarrold, M. F. Helix Formation in Unsolvated Alanine-Based Peptides: Helical Monomers and Helical Dimers. J. Am. Chem. Soc. 1999, 121, 3494-3501.

(4) Kohtani, M.; Jarrold, M. F. Water Molecule Adsorption on Short Alanine Peptides: How Short Is the Shortest Gas-Phase Alanine-Based Helix? J. Am. Chem. Soc. 2004, 126, 8454-8458.

(5) Chin, W.; Piuzzi, F.; Dognon, J.-P.; Dimicoli, I.; Tardivel, B.; Mons, M. Gas Phase Formation of a $310-$ Helix in a Three-Residue Peptide Chain: Role of Side Chain-Backbone Interactions as Evidenced by IR-UV Double Resonance Experiments. J. Am. Chem. Soc. 2005, 127, 11900-11901.

(6) Stearns, J. A.; Boyarkin, O. V.; Rizzo, T. R. Spectroscopic Signatures of Gas-Phase Helices: Ac-Phe-(Ala $)_{5}-\mathrm{Lys}^{-} \mathrm{H}^{+}$and Ac-Phe(Ala) ${ }_{10}$-Lys- $\mathrm{H}^{+}$. J. Am. Chem. Soc. 2007, 129, 13820-13821.

(7) Stearns, J. A.; Boyarkin, O. V.; Rizzo, T. R. Effects of N-Terminus Substitution on the Structure and Spectroscopy of Gas-Phase Helices. Chimia 2008, 62, 240-243.

(8) Stearns, J. A.; Seaiby, C.; Boyarkin, O. V.; Rizzo, T. R Spectroscopy and Conformational Preferences of Gas-phase Helices. Phys. Chem. Chem. Phys. 2009, 11, 125-132.

(9) Zabuga, A. V.; Rizzo, T. R. Capping Motif for Peptide Helix Formation. J. Phys. Chem. Lett. 2015, 6, 1504-1508.

(10) Rossi, M.; Blum, V.; Kupser, P.; von Helden, G.; Bierau, F.; Pagel, K.; Meijer, G.; Scheffler, M. Secondary Structure of AcAla $_{n} \mathrm{LysH}^{+}$Polyalanine Peptides $(\mathrm{n}=5,10,15)$ in Vacuo: Helical or Not? J. Phys. Chem. Lett. 2010, 1, 3465-3470.

(11) Schubert, F.; Pagel, K.; Rossi, M.; Warnke, S.; Salwiczek, M.; Koksch, B.; von Helden, G.; Blum, V.; Baldauf, C.; Scheffler, M. Native like Helices in a Specially Designed $\beta$ Peptide in the Gas Phase. Phys. Chem. Chem. Phys. 2015, 17, 5376-5385.

(12) Schubert, F.; Rossi, M.; Baldauf, C.; Pagel, K.; Warnke, S.; von Helden, G.; Filsinger, F.; Kupser, P.; Meijer, G.; Salwiczek, M.; et al. Exploring the Conformational Preferences of 20-residue Peptides in Isolation: $\mathrm{AcAla}_{19} \mathrm{LysH}^{+}$vs. AcLysAla ${ }_{19} \mathrm{H}^{+}$and the Current Reach of DFT. Phys. Chem. Chem. Phys. 2015, 17, 7373-7385. 
(13) Baldauf, C.; Rossi, M. Going Clean: structure and Dynamics of Peptides in the Gas Phase and Paths to Solvation. J. Phys.: Condens. Matter 2015, 27, 493002.

(14) Rossi, M.; Scheffler, M.; Blum, V. Impact of Vibrational Entropy on the Stability of Unsolvated Peptide Helices with Increasing Length. J. Phys. Chem. B 2013, 117, 5574-5584.

(15) Baldauf, C.; Hofmann, H.-J. Ab initio MO Theory - An Important Tool in Foldamer Research: Prediction of Helices in Oligomers of $\omega$-Amino Acids. Helv. Chim. Acta 2012, 95, 2348-2383.

(16) Rossi, M.; Chutia, S.; Scheffler, M.; Blum, V. Validation Challenge of Density-Functional Theory for Peptides - Example of AcPheAla ${ }_{5} \mathrm{LysH}^{+}$. J. Phys. Chem. A 2014, 118, 7349-7359.

(17) Hoffmann, W.; Marianski, M.; Warnke, S.; Seo, J.; Baldauf, C.; von Helden, G.; Pagel, K. Assessing the Stability of Alanine-based Helices by Conformer-selective IR Spectroscopy. Phys. Chem. Chem. Phys. 2016, 18, 19950-19954.

(18) Baldauf, C.; Pagel, K.; Warnke, S.; von Helden, G.; Koksch, B.; Blum, V.; Scheffler, M. How Cations Change Peptide Structure. Chem. - Eur. J. 2013, 19, 11224-11234.

(19) Ropo, M.; Schneider, M.; Baldauf, C.; Blum, V. First-principles Data Set of 45,892 Isolated and Cation-coordinated Conformers of 20 Proteinogenic Amino Acids. Sci. Data 2016, 3, 160009.

(20) Ropo, M.; Blum, V.; Baldauf, C. Trends for Isolated Amino Acids and Dipeptides: Conformation, Divalent Ion Binding, and Remarkable Similarity of Binding to Calcium and Lead. Sci. Rep. 2016, 6, 35772.

(21) De, S.; Musil, F.; Ingram, T.; Baldauf, C.; Ceriotti, M. Mapping and Classifying Molecules from a High-throughput Structural Database. J. Cheminf. 2017, 9, 6.

(22) Kohtani, M.; Kinnear, B. S.; Jarrold, M. F. Metal-Ion Enhanced Helicity in the Gas Phase. J. Am. Chem. Soc. 2000, 122, 12377-12378.

(23) Svendsen, A.; Lorenz, U. J.; Boyarkin, O. V.; Rizzo, T. R. A New Tandem Mass Spectrometer for Photofragment Spectroscopy of Cold, Gas-phase Molecular Ions. Rev. Sci. Instrum. 2010, 81, 073107.

(24) MacKerell, A. D.; Bashford, D.; Bellott, M.; Dunbrack, R. L.; Evanseck, J. D.; Field, M. J.; Fischer, S.; Gao, J.; Guo, H.; Ha, S.; et al. All-Atom Empirical Potential for Molecular Modeling and Dynamics Studies of Proteins. J. Phys. Chem. B 1998, 102, 3586-3616.

(25) Jorgensen, W. L.; Maxwell, D. S.; Tirado-Rives, J. Development and Testing of the OPLS All-Atom Force Field on Conformational Energetics and Properties of Organic Liquids. J. Am. Chem. Soc. 1996, $118,11225-11236$.

(26) Kaminski, G. A.; Friesner, R. A.; Tirado-Rives, J.; Jorgensen, W. L. Evaluation and Reparametrization of the OPLS-AA Force Field for Proteins via Comparison with Accurate Quantum Chemical Calculations on Peptides. J. Phys. Chem. B 2001, 105, 6474-6487.

(27) Wales, D. J.; Doye, J. P. K. Global Optimization by BasinHopping and the Lowest Energy Structures of Lennard-Jones Clusters Containing up to 110 Atoms. J. Phys. Chem. A 1997, 101, 5111-5116.

(28) Ponder, J. W.; Richards, F. M. An Efficient Newton-like Method for Molecular Mechanics Energy Minimization of Large Molecules. J. Comput. Chem. 1987, 8, 1016-1024.

(29) Ponder, J. W. TINKER - Software Tools for Molecular Design; Washington University School of Medicine: Saint Louis, MO, 1987.

(30) Blum, V.; Gehrke, R.; Hanke, F.; Havu, P.; Havu, V.; Ren, X.; Reuter, K.; Scheffler, M. Ab initio Molecular Simulations with Numeric Atom-centered Orbitals. Comput. Phys. Commun. 2009, 180, 2175-2196.

(31) Ren, X.; Rinke, P.; Blum, V.; Wieferink, J.; Tkatchenko, A.; Sanfilippo, A.; Reuter, K.; Scheffler, M. Resolution-of-identity Approach to Hartree-Fock, Hybrid Density Functionals, RPA, MP2 and GW with Numeric Atom-centered Orbital Basis Functions. New J. Phys. 2012, 14, 053020.

(32) Auckenthaler, T.; Blum, V.; Bungartz, H.-J.; Huckle, T.; Johanni, R.; Krämer, L.; Lang, B.; Lederer, H.; Willems, P. Parallel Solution of Partial Symmetric Eigenvalue Problems from Electronic Structure Calculations. Parallel Comput. 2011, 37, 783-794.

(33) Perdew, J.; Burke, K.; Ernzerhof, M. Generalized Gradient Approximation Made Simple. Phys. Rev. Lett. 1996, 77, 3865-3868.
(34) Tkatchenko, A.; Scheffler, M. Accurate Molecular van der Waals Interactions from Ground-State Electron Density and Free-Atom Reference Data. Phys. Rev. Lett. 2009, 102, 073005.

(35) Adamo, C.; Barone, V. Toward Reliable Density Functional Methods Without Adjustable Parameters: The PBE0Model. J. Chem. Phys. 1999, 110, 6158-6170.

(36) Ambrosetti, A.; Reilly, A. M.; DiStasio, R. A.; Tkatchenko, A. Long-range Correlation Energy Calculated from Coupled Atomic Response Functions. J. Chem. Phys. 2014, 140, 18 A508.

(37) Scott, A. P.; Radom, L. Harmonic Vibrational Frequencies: An Evaluation of Hartree-Fock, Møller-Plesset, Quadratic Configuration Interaction, Density Functional Theory, and Semiempirical Scale Factors. J. Phys. Chem. 1996, 100, 16502-16513.

(38) Merrick, J. P.; Moran, D.; Radom, L. An Evaluation of Harmonic Vibrational Frequency Scale Factors. J. Phys. Chem. A 2007, $111,11683-11700$.

(39) Alecu, I. M.; Zheng, J.; Zhao, Y.; Truhlar, D. G. Computational Thermochemistry: Scale Factor Databases and Scale Factors for Vibrational Frequencies Obtained from Electronic Model Chemistries. J. Chem. Theory Comput. 2010, 6, 2872-2887.

(40) Borukhov, I.; Andelman, D.; Orland, H. Adsorption of Large Ions from an Electrolyte Solution: A Modified Poisson-Boltzmann Equation. Electrochim. Acta 2000, 46, 221-229.

(41) Borukhov, I.; Andelman, D.; Orland, H. Steric Effects in Electrolytes: A Modified Poisson-Boltzmann Equation. Phys. Rev. Lett. 1997, 79, 435-438.

(42) Ringe, S.; Oberhofer, H.; Hille, C.; Matera, S.; Reuter, K. Function-Space-Based Solution Scheme for the Size-Modified PoissonBoltzmann Equation in Full-Potential DFT. J. Chem. Theory Comput. 2016, 12, 4052-4066.

(43) Voronina, L.; Masson, A.; Kamrath, M.; Schubert, F.; Clemmer, D.; Baldauf, C.; Rizzo, T. Conformations of Prolyl-Peptide Bonds in the Bradykinin 1-5 Fragment in Solution and in the Gas Phase. J. Am. Chem. Soc. 2016, 138, 9224-9233. 